\title{
Predictors of Mortality Using Machine Learning Decision Tree Algorithm in Critically III Adult Patients with COVID-19 Admitted to the ICU.
}

\section{Alyaa Elhazmi ( $\sim$ a.m.haz@live.com )}

Dr Sulaiman AlHabib Medical Center: Dr Sulaiman Al Habib Medical Group https://orcid.org/00000001-8315-7393

\section{Awad Alomari}

Dr Sulaiman AlHabib Medical Center: Dr Sulaiman Al Habib Medical Group

\section{Hend sallam}

King Faisal Centre Health Science Library: King Faisal Specialist Hospital and Research Center

Hani N Mufti

King Abdulaziz Medical City - Jeddah

Ahmed A. Rabie

King Saud Medical City

\section{Mohammed S Alshahrani}

King Fahd Hospital: King Fahad Hospital

\section{Ahmed Mady}

King Saud Medical City

\section{Adnan Alghamdi}

Prince Sultan Military Medical City

\section{Ali Altalaq}

Prince Sultan Military Medical City

\section{Mohamed H Azzam}

King Abdullah Medical Complex Jeddah

\section{Anees Sindi}

King Abdulaziz Medical City - Jeddah

\section{Ayman Kharaba}

King Fahad Hospital

\section{Zohair A Al-Aseri}

King Saud University College of Medicine

\section{Ghaleb A Almekhlafi}

Prince Sultan Military Medical City

\section{Wail Tashkandi}

King Abdulaziz University 


\section{Saud A Alajmi}

Prince Sultan Military Medical City

\section{Fahad Faqihi}

King Saud Medical City

\section{Abdulrahman Alharthy}

King Saud Medical City

Jaffar A Al-Tawfiq

Johns Hopkins: Johns Hopkins University

\section{Rami Ghazi Melibari}

king Abdullah Medical City

Waleed Al-Hazzani

McMaster University

Yaseen Arabi

King Abdulaziz University Faculty of Medicine

\section{Research}

Keywords: COVID-19, SARS-Cov2, decision tree, ICU, predictors, mortality.

Posted Date: October 29th, 2021

DOI: https://doi.org/10.21203/rs.3.rs-961542/v1

License: (c) (i) This work is licensed under a Creative Commons Attribution 4.0 International License. Read Full License 


\section{Abstract}

Background:

The Coronavirus Disease-19 (COVID-19) caused by the Severe Acute Respiratory Syndrome Coronavirus 2 (SARS-CoV-2) is a major cause of intensive care unit (ICU) admissions globally. Robust data of epidemiology, characteristics, and disease outcomes from different regions and populations showed considerable variations. However, limited number of reports addressed predictors of mortality utilizing machine learning methods. Herein, we aimed to describe the association and relationship of a predefined set of variables found to be predictive of 28-day ICU outcome among adults COVID-19 patients admitted to the ICU using a machine learning decision tree (DT) algorithm.

Methods:

This was a prospective/retrospective, multicenter cohort study from 14 hospitals in Saudi Arabia. We included critically ill COVID-19 patients admitted to the ICU between March 1, 2020, and October 31, 2020. The primary outcome was 28-day ICU mortality. Secondary outcomes were 90-day mortality and ICU length of stay. The predictors of mortality were identified using two predictive models, the conventional logistic regression and DT analysis.

Results:

A total of 1468 critically ill COVID-19 patients were included. The mean age was 55.9 (SD \pm 15.1 ) years, with $74 \%$ of the patients were males. The 28 -day ICU mortality was $540(36.8 \%)$, while 90 -day mortality was 600 (40.9\%). The multivariable logistic regression model demonstrated that the $\mathrm{PaO}_{2} / \mathrm{FiO}_{2}$ ratio on ICU admission and the need for intubation or vasopressors could strongly predict 28-day ICU mortality. The DT algorithm identified five variables [need for intubation, need for vasopressors, age, gender, and $\mathrm{PaO}_{2} / \mathrm{FiO}_{2}$ ratio] provided in an algorithmic fashion to predict 28-day ICU outcome.

\section{Conclusion:}

Five clinical predictors of 28-day ICU outcome were identified using DT algorithmic analysis of COVID-19 patients admitted to ICU. The findings of this DT analysis may be used in ICU for early identification of critically ill COVID-19 patients who are at high risk of 28-day mortality.

\section{Background}

The Coronavirus disease 2019 (COVID-19) caused by Severe Adult Respiratory Syndrome Coronavirus 2 (SARS-CoV-2) was first discovered in Wuhan city in late 2019 [1]. The World Health Organization (WHO) announced the disease to be pandemic on March 11, 2020 [2]. Since then, many investigators have addressed robust data of epidemiology, characteristics, and outcome of the disease from different regions and populations that showed variable results [3-8]. However, a limited number of reports addressed predictors of mortality utilizing machine learning methods [9]. Herein, we conducted this study 
to evaluate predictors of 28-day ICU mortality based on a predefined set of variables among COVID-19 adults admitted to the intensive care unit (ICU) using a machine learning decision tree (DT) algorithm.

\section{Methods}

\section{Study design}

This was a prospective/retrospective, multicenter national study. We included COVID-19 patients admitted to ICU between March 1, 2020, and October 31, 2020. Institutional review boards (IRB) approvals were obtained from the Central Institutional Review Board at the Saudi Ministry of Health and the Ethical Boards of each participating center.

\section{Setting}

The study was conducted in 14 participating centers across the Kingdom of Saudi Arabia. The participating ICUs were in accredited governmental and non-governmental tertiary hospitals. The multidisciplinary treating team included critical care physicians (consultants, specialists, and residents), registered ICU nurses, respiratory therapists, clinical pharmacists, and other ICU care providers who practiced according to national and international published protocols and guidelines. In addition, during the time of the COVID-19 surge, non- ICU physicians from different specialties joined the critical care team under the supervision of intensivists after receiving basic ICU management training.

\section{Patients}

Adult patients admitted to the ICU of participating hospitals with confirmed COVID-19 by detecting SARSCoV-2 using real-time polymerase chain reaction (RT-PCR) in nasopharyngeal swabs or tracheal aspirate specimens. Immunocompromised status was defined as solid organ malignancy, leukemia, current use of steroids (prednisone $>7 \mathrm{mg}$ daily for $>2$ weeks), post organ transplantation at any time, or rheumatological disease on immunomodulators (as azathioprine, methotrexate, infliximab, mycophenolate mofetil or others). Infection was defined by the positive culture in blood or tracheal aspirate.

\section{Data collection}

The data was collected using the Research Electronic Data Capture (REDCap, Vanderbilt University, Nashville, TN) [10]. The collected data included patients' demographics, comorbidities, signs, and symptoms of COVID-19 illness, laboratory abnormalities, mechanical ventilation (MV) utilization, adjunctive interventions, medications, complications, and outcomes.

\section{Outcomes measures}

The primary outcome was 28-day ICU mortality. Secondary outcomes were 90-day ICU mortality and hospital length of stay.

\section{Statistical analysis}




\section{General analysis}

Patients' characteristics were summarized using frequencies and percentages for categorical variables and as medians and interquartile ranges (IQR) or mean and standard deviation (SD) for continuous variables. Chi-square or Fisher's exact tests compared categorical variables. The Wilcoxon rank-sum test was used for continuous variables. We constructed Kaplan-Meier curves to assess cumulative mortality during the initial 60 days from ICU admission.

In the first 28 days of ICU stay, risk factors of mortality were evaluated in the whole cohort using univariate and multivariable logistic regression analyses. Variables included in the multivariable logistic regression model were identified based on literature review and used to generate the predictive models included demographics, co-morbid conditions, laboratory data on ICU admission, the respiratory component of SOFA score [11] and the need for intubation or vasopressors. In the regression analysis, variables of the need for intubation and vasopressors included patients during the first five days of ICU admission, and the respiratory component of SOFA score on ICU admission was classified as (category 4 was $\mathrm{PaO}_{2} / \mathrm{FiO}_{2}<100$ with respiratory support, while category 0-3 was $\mathrm{PaO}_{2} / \mathrm{FiO}_{2}>100$ ). Continuous variables were categorized using cut-off points based on either previous literature review or optimal cutoff points statistically identified using the cut pointer library in R, maximizing the Youdin index that determines the split point between survivors and non-survivors. The logistic regression model results were reported as odds ratio $(\mathrm{OR})$ with a $95 \%$ confidence interval $(95 \% \mathrm{Cl})$. All statistical tests were two-tailed, and $p$ values $<0.05$ were considered significant. All statistical analyses were performed using $R$ software, version 4.0.2 (06-22-2020) (R Foundation for Statistical Computing, Vienna, Austria).

\section{Decision Tree (DT) analysis}

Machine learning DT analysis was used to identify characteristics of patients with COVID-19 that were predictive of 28-day ICU outcome. The model was generated using the standard setting in the opensource software library Waikato Environment for Knowledge Analysis (WEKA, University of Waikato) [12] using the $\mathrm{C} 4.5$ classification algorithm (J48) with 20 cases as the minimum number of cases at the leaf of each branch (end of the tree). C4.5 classifier used the information gain ratio split criterion to reduce bias towards multivalued [13]. In addition, we used "Algorithm Accuracy" as a general measure to assess the performance of the classifier. Accuracy is one of the most used performance measures representing the overall correctness of the algorithm. The Area Under the Receiver Operating Characteristic Curve (AUROC) is also used to evaluate the performance of the decision tree model, which represents the predictive performance of the model across different thresholds of sensitivity (true positive rate (TPR)) plotted over different ranges of 1- specificity (false positive rate) [14]. Ten-fold cross validation was applied to generate the accuracy, AUROC, and its confidence intervals. TPR is the true positive cases by the algorithm divided by the total positive cases (true positive + false positive). To assess the model's generalizability and avoid over-fitting, 10 -folds cross validation was applied.

\section{Results}




\section{Patient characteristics and ICU admission data}

During the study period, 1468 patients were admitted to the ICUs in the 14 participating hospitals. Table 1 described the patients' demographics and data in the first 24 hours of ICU admission among the 28-day ICU survivors vs. non-survivors. The mean age was 55.9 (SD \pm 15.1$)$ years, $(74 \%)$ of the patients were males, and 69 patients (4.8\%) were healthcare workers. Hypertension, ischemic heart disease, and smoking were significantly associated with higher 28-day ICU mortality (P value $0.0187,0.0016$, and 0.0333 , respectively). The SOFA score, 7 (IQR 4-10), was significantly higher in patients who died within the first 28 days of ICU admission. Survivors had a higher $\mathrm{PaO}_{2} / \mathrm{FiO}_{2}$ ratio compared to non-survivors at 28-day of ICU admission [142 (IQR 72-176) vs. 92 (IQR 66-138), p value <0.001]. (Table 1) 
Table 1

Baseline general characteristics and ICU admission data.

\begin{tabular}{|c|c|c|c|c|}
\hline Patient characteristic & $\begin{array}{l}\text { All Patients } \\
(\mathrm{n}=1468) \\
\text { /Denominator }\end{array}$ & $\begin{array}{l}\text { 28-day } \\
\text { non- } \\
\text { survivors } \\
(n=540)\end{array}$ & $\begin{array}{l}28-d a y \\
\text { survivors } \\
(n=928)\end{array}$ & $p$-value \\
\hline Age (years), mean ( $\pm S D)$ & $55.9(15.1) / 1423$ & $58.4(15.2)$ & $54.3(14.9)$ & $<0.001^{*}$ \\
\hline \multicolumn{5}{|l|}{ Gender, n (\%) } \\
\hline Male gender, n (\%) & $1085(74) / 1467$ & $413(76.6)$ & $672(72.4)$ & 0.0765 \\
\hline Female Gender, n (\%) & $382(26) / 1467$ & $126(23.4)$ & $256(27.6)$ & \\
\hline Pregnancy, n (\%) & $19(5.1) / 372$ & $2(1.6)$ & $17(6.8)$ & $0.0425^{\star}$ \\
\hline Healthcare worker, n (\%) & $69(4.8) / 1436$ & $15(2.9)$ & $54(5.9)$ & 0.009 * \\
\hline $\mathrm{BMI}\left(\mathrm{kg} / \mathrm{m}^{2}\right)$, mean $( \pm \mathrm{SD})$ & $30.1(6.8) / 1369$ & $29.5(6.5)$ & $30.5(7)$ & 0.009 * \\
\hline \multicolumn{5}{|l|}{ Comorbidity } \\
\hline Diabetes mellitus, n (\%) & $770(54.8) / 1405$ & $297(58.1)$ & $473(52.9)$ & 0.0589 \\
\hline Hypertension, n (\%) & $676(48.6) / 1391$ & $267(52.8)$ & $409(46.2)$ & $0.0187 *$ \\
\hline Ischemic heart disease, n (\%) & $184(13.8) / 1333$ & $85(17.8)$ & 99 (11.6) & $0.0016 *$ \\
\hline Bronchial Asthma, n (\%) & $128(9.6) / 1333$ & $37(7.7)$ & $91(10.6)$ & 0.0845 \\
\hline Chronic Kidney Disease, n (\%) & $123(9.2) / 1339$ & $47(9.7)$ & $76(8.9)$ & 0.656 \\
\hline Smoker, n (\%) & $85(7.2) / 1180$ & $21(5)$ & $64(8.4)$ & $0.0333^{*}$ \\
\hline Left ventricular failure, n (\%) & $74(5.6) / 1331$ & $32(6.7)$ & $42(4.9)$ & 0.181 \\
\hline Immunocompromised, n (\%) & $72(5.4) / 1332$ & $19(4)$ & $53(6.2)$ & 0.079 \\
\hline $\begin{array}{l}\text { Renal Replacement therapy, } \mathrm{n} \\
(\%)\end{array}$ & $54(4.1) / 1332$ & $25(5.2)$ & $29(3.4)$ & 0.12 \\
\hline Cancer, n (\%) & $48(3.6) / 1333$ & $17(3.5)$ & $31(3.6)$ & 0.913 \\
\hline Chronic Lung Disease, n (\%) & $38(2.9) / 1327$ & $14(2.9)$ & $24(2.8)$ & 0.891 \\
\hline Solid-organ transplant, n (\%) & $29(2.2) / 1334$ & $8(1.7)$ & $21(2.5)$ & 0.337 \\
\hline COPD, n (\%) & $26(2) / 1327$ & $12(2.5)$ & $14(1.6)$ & 0.27 \\
\hline
\end{tabular}

COPD, chronic obstructive pulmonary disease. BMI, body mass index. COPD, chronic obstructive pulmonary disease. CRP, C - reactive protein. GCS, Glasgow coma scale. HR, heart rate. LDH, Lactic Acid Dehydrogenase. MAP mean arterial pressure. NL ratio, Neutrophil-to-lymphocyte ratio. RR, respiratory rate. SOFA, Sequential Organ Failure Assessment. WBC, white blood cells. 


\begin{tabular}{|c|c|c|c|c|}
\hline Patient characteristic & $\begin{array}{l}\text { All Patients } \\
(n=1468) \\
\text { /Denominator }\end{array}$ & $\begin{array}{l}\text { 28-day } \\
\text { non- } \\
\text { survivors } \\
(n=540)\end{array}$ & $\begin{array}{l}28 \text {-day } \\
\text { survivors } \\
(n=928)\end{array}$ & $p$-value \\
\hline Chronic Liver Disease, n (\%) & $24(1.8) / 1332$ & $10(2.1)$ & $14(1.6)$ & 0.557 \\
\hline $\begin{array}{l}\text { Chronic Hematological Disease } \\
(\%)\end{array}$ & $12(0.9) / 1337$ & $3(0.6)$ & $9(1.1)$ & 0.553 \\
\hline \multicolumn{5}{|l|}{ ICU admission data (first 24 hs) } \\
\hline Use of inotropes, n (\%) & $189(15.3) / 1235$ & $120(29.4)$ & $69(8.3)$ & $<0.001^{*}$ \\
\hline $\begin{array}{l}\text { New AKI on ICU admission, } \mathrm{n} \\
(\%)\end{array}$ & $98(8.6) / 1137$ & $60(14.4)$ & $38(5.3)$ & $<0.001^{*}$ \\
\hline MAP $(\mathrm{mmHg})$, mean $( \pm \mathrm{SD})$ & $86.1(16.1) / 1299$ & $84.7(16.7)$ & $86.8(16.3)$ & $0.0246^{\star}$ \\
\hline HR (beat/min), mean $( \pm S D)$ & $91.2(20.4) / 1294$ & $95.1(20.7)$ & $89.3(20)$ & $<0.001^{*}$ \\
\hline RR (per min), mean ( $\pm S D)$ & $27.9(7.2) / 1274$ & $28.3(7.1)$ & $27.8(7.3)$ & 0.196 \\
\hline GCS, median $( \pm \mid \mathrm{QR})$ & $15(14-15) / 1259$ & $15(9-15)$ & $15(15-15)$ & $<0.001^{*}$ \\
\hline SOFA, median $( \pm I Q R)$ & $4(3-8) / 1333$ & $7(4-10)$ & $4(2-6)$ & $<0.001^{*}$ \\
\hline $\mathrm{PO}_{2} / \mathrm{FiO}_{2}$ ratio, median (IQR) & $134(71-163) / 1217$ & $92(66-138)$ & $142(72-176)$ & $<0.001^{*}$ \\
\hline $\mathrm{PO}_{2} / \mathrm{FiO}_{2}$ ratio, $\mathrm{n}(\%)$ & & & & $<0.001^{\star}$ \\
\hline$<100$ & $550(50) / 1099$ & $210(57.9)$ & $340(46.2)$ & \\
\hline $100-<200$ & $361(32.8) / 1099$ & $110(30.3)$ & $251(34.1)$ & \\
\hline $200-300$ & $116(10.6) / 1099$ & $27(7.4)$ & $89(12.1)$ & \\
\hline$>300$ & $72(6.6) / 1099$ & $16(4.4)$ & $56(7.6)$ & \\
\hline \multicolumn{5}{|c|}{ ICU admission laboratory data (first 24 hs) } \\
\hline WBC $(x 109 / L)$, mean $( \pm S D)$ & $10.7(6.3) / 1378$ & $12.3(7.8)$ & $9.7(5)$ & $<0.001^{*}$ \\
\hline NL Ratio, mean $( \pm S D)$ & $10.3(8.7) / 1126$ & $11.8(9.4)$ & $9.4(8.1)$ & $<0.001^{*}$ \\
\hline $\begin{array}{l}\text { Creatinine }(\mathrm{nmol} / \mathrm{L}) \text {, median } \\
\text { (IQR) }\end{array}$ & $83(63-130) / 1289$ & $106(71-187)$ & $75(60-105)$ & $<0.001^{*}$ \\
\hline $\begin{array}{l}\text { Lactate }(\mathrm{mmol} / \mathrm{L}) \text {, median } \\
(\mathrm{IQR})\end{array}$ & $1.5(1.1-2.2) / 652$ & $1.8(1.2-3)$ & $1.5(1.1-2)$ & $<0.001^{*}$ \\
\hline
\end{tabular}

COPD, chronic obstructive pulmonary disease. BMI, body mass index. COPD, chronic obstructive pulmonary disease. CRP, C - reactive protein. GCS, Glasgow coma scale. HR, heart rate. LDH, Lactic Acid Dehydrogenase. MAP mean arterial pressure. NL ratio, Neutrophil-to-lymphocyte ratio. RR, respiratory rate. SOFA, Sequential Organ Failure Assessment. WBC, white blood cells. 


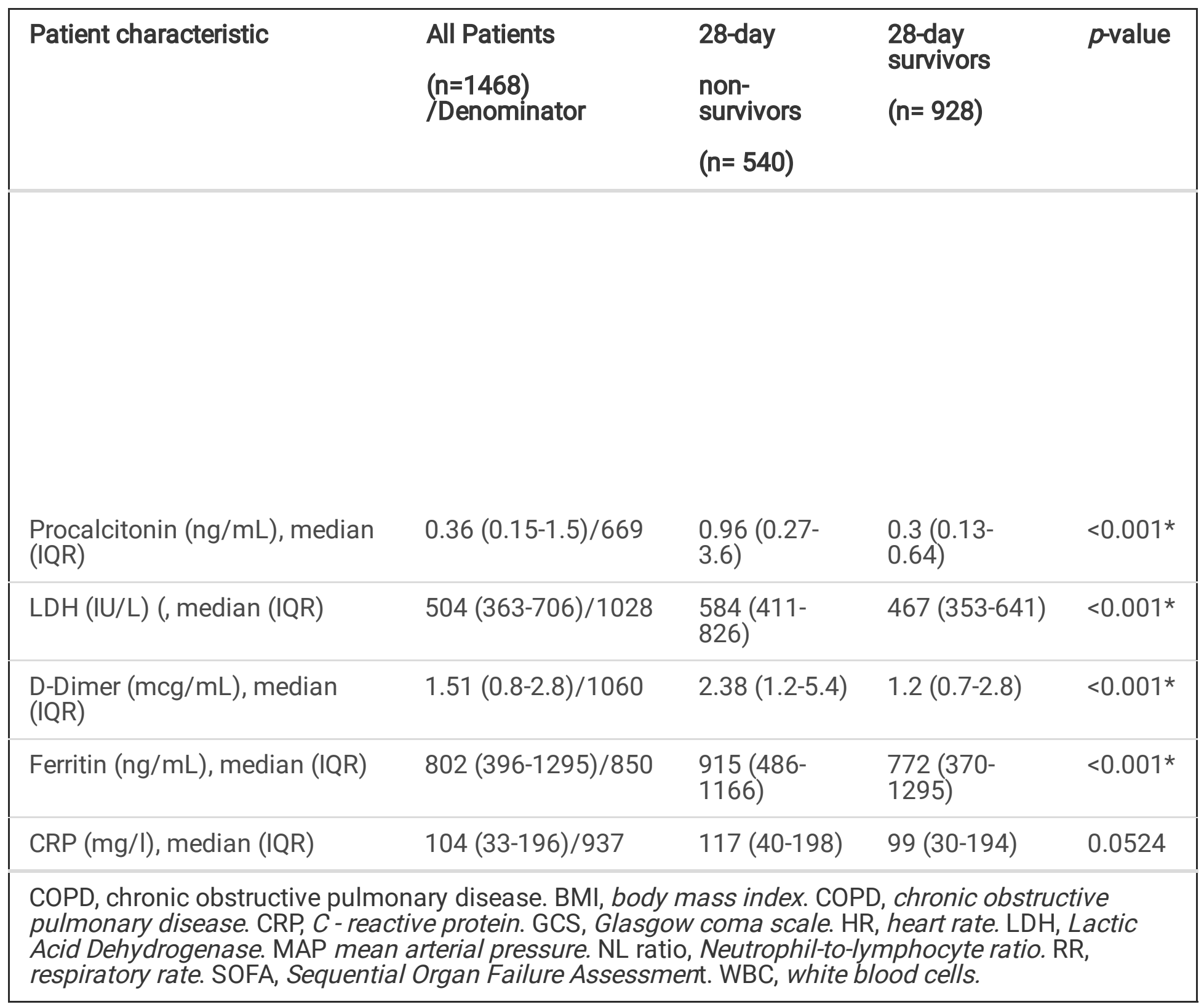

\section{Interventions during ICU stay}

Out of the study cohort, 778 patients $(52.9 \%)$ required invasive mechanical ventilation (IMV) during the ICU admission, of whom 128 patients were intubated prior to transfer to the ICU of the participating centers. High flow nasal cannula (HFNC) was used in 446 patients (35.3\%) with median duration of 3 (IQR 2-6) days. In non-intubated patients, awake prone position was utilized in 350 patients (27.7\%), of whom 194 patients $(57.4 \%)$ utilized it for a duration longer than 4 hours/day. Of patients who required IMV, 506 patients $(75.3 \%)$ received neuromuscular blockade, and 319 patients (47.8\%) received prone positioning (Table 2). 
Table 2

Interventions during ICU stay.

\begin{tabular}{|c|c|c|c|c|}
\hline Patient characteristic & $\begin{array}{l}\text { All Patients }(n=1468) \\
\text { /Denominator }\end{array}$ & $\begin{array}{l}\text { 28-day } \\
\text { non- } \\
\text { survivors } \\
(n=540)\end{array}$ & $\begin{array}{l}\text { 28-day } \\
\text { survivors } \\
(n=928)\end{array}$ & $p$-value \\
\hline Vasopressors n (\%) & $395(26.9) / 1468$ & $246(45.5)$ & $149(16)$ & $<0.001^{*}$ \\
\hline \multicolumn{5}{|l|}{ Oxygen delivery modes } \\
\hline HFNC, n (\%) & $446(35.3) / 1264$ & $105(25.1)$ & $341(40.4)$ & $<0.001^{*}$ \\
\hline HFNC days, median (IQR) & $3(2-6) / 429$ & $2(1-4)$ & $4(2-6)$ & $<0.001^{*}$ \\
\hline NIPPV, n (\%) & $205(16.2) / 1267$ & $96(22.8)$ & 109 (12.9) & $<0.001^{*}$ \\
\hline NIPPV days, median (IQR) & $2(1-4) / 198$ & $2(1-4)$ & $2(2-6)$ & 0.527 \\
\hline $\begin{array}{l}\text { Awake prone positioning, } n \\
(\%)\end{array}$ & $350(27.7) / 1263$ & $86(20.6)$ & $264(31.2)$ & $<0.001^{*}$ \\
\hline $\begin{array}{l}\text { Awake prone days, median } \\
\text { (IQR) }\end{array}$ & $3(2-5) / 316$ & $2(1-4)$ & $4(2-6)$ & $<0.001^{*}$ \\
\hline $\begin{array}{l}\text { Awake prone }>4 \text { hours/day, } \\
n(\%)\end{array}$ & $194(57.4) / 338$ & $\begin{array}{l}49 \\
(60.5) / 81\end{array}$ & $145(56.4)$ & 0.518 \\
\hline IMV, n (\%) & $778(52.9) / 1468$ & $454(83.9)$ & $32434.9)$ & $<0.001^{*}$ \\
\hline \multicolumn{5}{|l|}{$\begin{array}{l}\text { First } 24 \text { hs of intubation, } \\
\text { mean }( \pm S D)\end{array}$} \\
\hline $\mathrm{PaO}_{2} / \mathrm{FiO}_{2}$ & $125.5(80) / 535$ & $115(76)$ & $137.7(83)$ & $0.001^{*}$ \\
\hline $\mathrm{PCO}_{2}(\mathrm{mmHg})$ & $46.6(14.7) / 558$ & $48(15.5)$ & $45(13.5)$ & $0.015^{\star}$ \\
\hline $\begin{array}{l}\text { Static Compliance } \\
\left(\mathrm{ml} / \mathrm{cmH}_{2} \mathrm{O}\right)\end{array}$ & $27.8(11.2) / 152$ & $27(11.7)$ & $28.6(10.6)$ & 0.382 \\
\hline 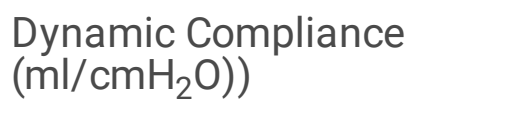 & $22.2(14.6) / 324$ & $20.6(9.4)$ & $24.1(19.2)$ & $0.045^{\star}$ \\
\hline $\begin{array}{l}\text { Peak airway pressure } \\
\left(\mathrm{cmH}_{2} \mathrm{O}\right)\end{array}$ & $31.2(6.7) / 335$ & $31.7(7.1)$ & $30.6(6.2)$ & 0.131 \\
\hline Plateau pressure $\left(\mathrm{cmH}_{2} \mathrm{O}\right)$ & $27.3(5.7) / 156$ & $27(5.4)$ & $27.5(5.9)$ & 0.549 \\
\hline
\end{tabular}

HFNC, high flow nasal cannula. IVIG, Intravenous immunoglobulin. NIPPV, non-invasive positive pressure ventilation. IMV, Invasive Mechanical Ventilation. $\mathrm{PaO}_{2} / \mathrm{FiO}_{2}$, Partial pressure of oxygen to fraction of inspired oxygen ratio. PCO2, Partial pressure of Co2. Fio2, fraction of inspired oxygen. $\mathrm{MN}$, mechanical ventilator. APRV, Airway pressure release ventilation. ECMO, extracorporeal membrane oxygenation. HFOV, high-frequency oscillatory ventilation. iNO, inhaled nitric oxide. 


\begin{tabular}{|c|c|c|c|c|}
\hline Patient characteristic & $\begin{array}{l}\text { All Patients }(n=1468) \\
\text { /Denominator }\end{array}$ & $\begin{array}{l}\text { 28-day } \\
\text { non- } \\
\text { survivors } \\
(n=540)\end{array}$ & $\begin{array}{l}\text { 28-day } \\
\text { survivors } \\
(n=928)\end{array}$ & $p$-value \\
\hline Tidal Volume (ml per IBW) & $6.94(1.34) / 601$ & $6.87(1.2)$ & $7.04(1.51)$ & 0.135 \\
\hline \multicolumn{5}{|l|}{$\begin{array}{l}\text { Interventions during IMV, n } \\
(\%)\end{array}$} \\
\hline $\begin{array}{l}\text { Neuromuscular blockade } \\
\text { infusion }\end{array}$ & $562(74.8) / 751$ & $332(76.1)$ & $230(73)$ & 0.329 \\
\hline Recruitment maneuvers use & $91(12.3) / 737$ & $50(11.6)$ & $41(13.4)$ & 0.482 \\
\hline iNO use & $64(8.6) / 742$ & $37(8.5)$ & $27(8.7)$ & 0.927 \\
\hline Prone positioning during $\mathrm{MV}$ & $350(47) / 745$ & $194(44.6)$ & $156(50.3)$ & 0.123 \\
\hline Rescue APRV use & $20(2.7) / 739$ & $10(2.3)$ & $10(3.2)$ & 0.444 \\
\hline Rescue HFOV use & $12(1.6) / 740$ & $8(1.8)$ & $4(1.3)$ & 0.563 \\
\hline Tracheostomy & $60(7.7) / 778$ & $8(1.5)$ & $52(5.6)$ & $\begin{array}{l}<0.001 \\
\star\end{array}$ \\
\hline ECMO & $71(9.1) / 778$ & $40(7.5)$ & $31(3.4)$ & $\underset{\star}{<0.001}$ \\
\hline \multicolumn{5}{|l|}{$\begin{array}{l}\text { Medications and } \\
\text { interventions, } \mathrm{n}(\%)\end{array}$} \\
\hline Azithromycin & $1069(74.2) / 1440$ & $366(68.9)$ & 703 (77.3) & $<0.001 *$ \\
\hline Corticosteroids & $1048(73.1) / 1433$ & $401(75.8)$ & $647(71.6)$ & 0.081 \\
\hline Chloroquine & $429(30.5) / 1406$ & $152(29.2)$ & 277 (31.3) & 0.403 \\
\hline Tocilizumab & $426(30.1) / 1414$ & $126(24)$ & $300(33.7)$ & $<0.001 *$ \\
\hline Favipiravir & $316(22.4) / 1441$ & $111(21.2)$ & $205(23.1)$ & 0.401 \\
\hline Ribavirin & $241(17.2) / 1402$ & 79 (15.3) & $162(18.3)$ & 0.141 \\
\hline Convalescent plasma & $53(3.8) / 1409$ & $12(2.3)$ & $41(4.6)$ & $0.026^{\star}$ \\
\hline IVIG & $51(3.6) / 1401$ & $18(3.5)$ & $33(3.7)$ & 0.792 \\
\hline Plasmapheresis & $26(1.8) / 1409$ & $17(3.2)$ & $9(1)$ & $0.002^{\star}$ \\
\hline \multicolumn{5}{|c|}{$\begin{array}{l}\text { HFNC, high flow nasal cannula. IVIG, Intravenous immunoglobulin. NIPPV, non-invasive positive } \\
\text { pressure ventilation. IMV, Invasive Mechanical Ventilation. } \mathrm{PaO}_{2} / \mathrm{FiO}_{2}, \text { Partial pressure of oxygen to } \\
\text { fraction of inspired oxygen ratio. PCO2, Partial pressure of } \mathrm{Co}_{2} \text {. } \mathrm{Fio} 2 \text {, fraction of inspired oxygen. } \mathrm{MN} \text {, } \\
\text { mechanical ventilator. APRV, Airway pressure release ventilation. ECMO, extracorporeal membrane } \\
\text { oxygenation. HFOV, high-frequency oscillatory ventilation. iNO, inhaled nitric oxide. }\end{array}$} \\
\hline
\end{tabular}




\begin{tabular}{|c|c|c|c|c|}
\hline Patient characteristic & $\begin{array}{l}\text { All Patients }(n=1468) \\
\text { /Denominator }\end{array}$ & $\begin{array}{l}\text { 28-day } \\
\text { non- } \\
\text { survivors } \\
(n=540)\end{array}$ & $\begin{array}{l}\text { 28-day } \\
\text { survivors } \\
(n=928)\end{array}$ & $p$-value \\
\hline Remdesivir & $13(0.9) / 1399$ & $5(1)$ & $8(0.9)$ & 0.91 \\
\hline \multicolumn{5}{|c|}{$\begin{array}{l}\text { HFNC, high flow nasal cannula. IVIG, Intravenous immunoglobulin. NIPPV, non-invasive positive } \\
\text { pressure ventilation. IMV, Invasive Mechanical Ventilation. } \mathrm{PaO}_{2} / \mathrm{FiO}_{2} \text {, Partial pressure of oxygen to } \\
\text { fraction of inspired oxygen ratio. } \mathrm{PCO} 2 \text {, Partial pressure of } \mathrm{Co}_{2} \text {. Fio2, fraction of inspired oxygen. MN, } \\
\text { mechanical ventilator. APRV, Airway pressure release ventilation. ECMO, extracorporeal membrane } \\
\text { oxygenation. } \mathrm{HFOV} \text {, high-frequency oscillatory ventilation. iNO, inhaled nitric oxide. }\end{array}$} \\
\hline
\end{tabular}

\section{Outcomes:}

Out of 1468 patients, 540 (36.8\%) died within 28-day of ICU stay, while 757 (51.6\%) were discharged alive from the hospital. The 90-day ICU mortality was 600 patients (40.9\%), and the median ICU length of stay was 9 (IQR 5-16) days, while the hospital length of stay was 15 (IQR 9-24) days. Blood cultures were positive in 267 patients (24.3\%) and respiratory samples in 227 patients (33.4\%) (Table 3). The Kaplan Maier curve for COVID-19 cumulative incidence of mortality showed $40 \%$ mortality at day 60 of ICU admission. (Figure 1) 
Table 3

Patients Outcomes

\begin{tabular}{|c|c|c|c|c|}
\hline & $\begin{array}{l}\text { All Patients } \\
(n=1468) \\
\text { /Denominator }\end{array}$ & $\begin{array}{l}\text { 28-day } \\
\text { non- } \\
\text { survivors } \\
(n=540)\end{array}$ & $\begin{array}{l}\text { 28-day } \\
\text { survivors } \\
(n=928)\end{array}$ & $\begin{array}{l}p- \\
\text { value }\end{array}$ \\
\hline ICU Mortality at 28 days, $\mathrm{n}(\%)$ & $540(36.8)$ & & & \\
\hline 90-day mortality, n (\%) & $600(40.9)$ & & & \\
\hline Discharge from ICU alive, n (\%) & $869(59.1)$ & & & \\
\hline Discharged from hospital alive, $\mathrm{n}(\%)$ & $757(51.6)$ & & & \\
\hline Transfer to another institution, $\mathrm{n}(\%)$ & $94(6.5)$ & & & \\
\hline \multicolumn{5}{|l|}{$\begin{array}{l}\text { Mortality Subgroup analysis according to } \\
\text { time of death }\end{array}$} \\
\hline $0-28$ days, $\mathrm{n}(\%)$ & $540(90.05)$ & & & \\
\hline $29-60$ days, $n(\%)$ & $54(8.9)$ & & & \\
\hline $61-90$ days, $\mathrm{n}(\%)$ & $6(0.06)$ & & & \\
\hline ICU length of stay (days), Median (IQR) & $9(5-16) / 1468$ & $10(5-16)$ & $9(5-17)$ & 0.356 \\
\hline $\begin{array}{l}\text { Hospital length of stay, (days) Median } \\
\text { (IQR) }\end{array}$ & $15(9-24) / 1468$ & $13(7-18)$ & $18(11-31)$ & $\begin{array}{l}<0.001 \\
\star\end{array}$ \\
\hline $\begin{array}{l}\text { Mechanical ventilation duration (days) } \\
\text { Median (IQR) }\end{array}$ & $7(0-14) / 1082$ & $10(4-15)$ & $3(0-11)$ & $\begin{array}{l}<0.001 \\
\star\end{array}$ \\
\hline $\begin{array}{l}\text { Infection (positive culture, respiratory), n } \\
(\%)\end{array}$ & $227(33.4) / 679$ & $\begin{array}{l}131 \\
(46.6)\end{array}$ & $96(24.1)$ & $\begin{array}{l}<0.001 \\
\star\end{array}$ \\
\hline Infection (positive culture, blood), n (\%) & $267(24.3) / 1100$ & $\begin{array}{l}151 \\
(34.2)\end{array}$ & $116(17.6)$ & $\begin{array}{l}<0.001 \\
*\end{array}$ \\
\hline $\mathrm{AKI}$ requiring $\mathrm{RRT}, \mathrm{n}(\%)$ & $178(14.1) / 1259$ & $\begin{array}{l}130 \\
(28.6)\end{array}$ & $48(6)$ & $\begin{array}{l}<0.001 \\
\star\end{array}$ \\
\hline Pneumothorax, n (\%) & $89(6.2) / 1440$ & $55(10.4)$ & $34(3.7)$ & $\begin{array}{l}<0.001 \\
\star\end{array}$ \\
\hline Myocardial infarction, n (\%) & $64(4.5) / 1433$ & $40(7.6)$ & $24(2.6)$ & $<0.001$ \\
\hline GI Bleeding, n (\%) & $52(3.6) / 1438$ & $27(5.1)$ & $25(2.7)$ & 0.019 \\
\hline Pulmonary embolism, n (\%) & $44(3.1) / 1435$ & $20(3.8)$ & $24(2.6)$ & 0.215 \\
\hline
\end{tabular}

AKI, acute kidney injury ICU, intensive care unit. GI, gastrointestinal. RRT, renal replacement therapy. 


\begin{tabular}{|c|c|c|c|c|}
\hline & $\begin{array}{l}\text { All Patients } \\
\text { (n=1468) } \\
\text { /Denominator }\end{array}$ & $\begin{array}{l}\text { 28-day } \\
\text { non- } \\
\text { survivors } \\
(n=540)\end{array}$ & $\begin{array}{l}\text { 28-day } \\
\text { survivors } \\
(n=928)\end{array}$ & $\begin{array}{l}p- \\
\text { value }\end{array}$ \\
\hline Deep vein thrombosis, $\mathrm{n}(\%)$ & $33(2.3) / 1435$ & $17(3.2)$ & $16(1.8)$ & 0.070 \\
\hline Intracranial hemorrhage, n (\%) & $33(2.3) / 1432$ & $15(2.9)$ & $18(2)$ & 0.277 \\
\hline Ischemic Stroke, n (\%) & $32(2.2) / 1438$ & $14(2.7)$ & $18(2)$ & 0.39 \\
\hline
\end{tabular}

\section{Predictors of 28-day ICU mortality}

Results of the logistic regression:

The multivariable logistic regression analysis retained the respiratory component of SOFA score (category 4) and the need for intubation and vasopressors as predictors of 28-day ICU mortality [(OR 2.12 (95\% Cl 1.21-3.7), $\mathrm{P}$ value $<0.008)$, (OR 3.25 ( $95 \% \mathrm{Cl} 1.76-5.99)$, $\mathrm{P}$ value $<0.001)$; and (OR 3.11 (95\% $\mathrm{Cl} 1.66-5.82), \mathrm{P}$ value $<0.001)$ ] respectively. (Table 4 )

The stepwise logistic regression analysis retained eight variables: age groups, gender, diabetes mellitus (DM), ischemic heart disease (IHD), the respiratory component of SOFA score (category 4), need for intubation, or vasopressors, and neutrophils-lymphocytes $(\mathrm{NL})$ ratio. (Figure 3)

Results of the decision tree analysis:

Five variables were identified and allocated the patients into the final binary outcome (Survival versus Mortality). These variables were: the need for intubation or vasopressors, Gender, $\mathrm{PaO}_{2} / \mathrm{FiO}_{2}$ on ICU admission, and age categories. The constructed DT assigned the root node (start of the tree or first splitting criteria) to the need for intubation. Then, the tree continued to grow and assign patients into their respective groups utilizing the other four variables in a sequential manner. The DT model's ability to correctly assign patients to their respective groups (model discrimination), assessed using the ROC-AUC, was $75.42 \%$ (95\% Cl= 74.84-78.95). The DT model accuracy was $73.1 \%$ (number of retained patients on the model 1043 out of 1468). (Figure 2).

\section{Discussion}

We utilized the DT analysis and identified five features that are predictive of 28-day ICU outcomes. These features are the need for intubation, vasopressors, age, gender, and $\mathrm{PaO}_{2} / \mathrm{FiO}_{2}$ ratio. 
The COVID-19 pandemic overwhelmed the health care system leading to constraints of medical resources, mainly in critical care unit capacity, and a shortage of mechanical ventilators. [15-17]. Many hospitals utilized machine learning-based analyses combining clinical, radiological and laboratory data for the prognostication and rapid risk stratification of PCR confirmed COVID-19 patients [18-20]. The evaluation of the severity of illness for patients admitted to the ICU has been applied by different general scoring methods such as the acute physiology and chronic health evaluation (APACHE) II and IV [21, 22], Simplified Acute Physiology Score (SAPS) [23], and SOFA scores [11] or COVID-19 specific scores as 4C mortality score [24]. APACHE-II score, the most commonly utilized score on ICU admission, is subjective, time-consuming, and depends mainly on laboratory indicators which are not comprehensive enough to predict the outcome of the newly emerged COVID-19 [25]. Standard logistic regression analysis is useful in predicting outcomes of interest; however, it does not model a nonlinear relationship of multiple dimensional data [26].

The application of machine-learning models in the medical field has been increasingly utilized, especially in outcome prediction of cancer outcomes [27-29]. Random Forest classifiers, decision trees, and artificial neural networks (ANNs) specifically were among the earliest used techniques in medical research $[30,31]$. DT analysis is an effective classifier that has been applied in many domains $[32,33]$ and is considered an intuitive nonlinear approach that can automatically detect independent variables that predict outcomes and the interactions between these variables. Moreover, it allows an easy-to-understand visual representation of the relationships between the variables and the primary outcome [34]. The multivariable, stepwise logistic regression, and DT analyses in our study were built using the same predefined set of variables. Nevertheless, our study demonstrates the advantage of DT analysis in providing prediction in an algorithmic fashion rather than merely identifying associations and relations between the variables and the outcome achieved by most regression models [35, 36]. In this context, DT algorithms are very intuitive, easy to understand and explain while producing simple rules that simulate a human logic-like approach.

Predictors of mortality in COVID-19 have been widely reported in many studies with different settings and designs, including mainly laboratory and radiological variables [37, 38]. Limited reports, however, studied clinical variables on ICU admission as predictors of mortality that may facilitate the early identification of critically ill COVID-19 patients at higher risk of 28-day mortality [39]. In metanalysis reported by (Pengqiang Du. et al. 2021) addressed the predictors of mortality utilizing the classic logistic regression analysis showed that advanced age, male gender, and comorbidities of chronic respiratory disease, DM, hypertension, and chronic kidney or cardiovascular diseases were associated with severe illness or death for COVID-19 patients [40].

On the other hand, studies that reported predictors of mortality utilizing DT analysis in critically ill COVID19 patients were very limited [41-43]. One of these analyses by (Qiao Yang et al. 2021) showed a rapid, simple, and easy to interpret decision tree model built of three biochemical markers on ICU admission (LDH, NLR, and CRP) with a high true sensitivity rate to predict death in severe COVID-19 disease [41]. 
Strengths of the study include the multicenter nature, which improves generalizability. In addition, unlike the earlier reported experience from the Middle East [44], the 28-day ICU mortality of $36.8 \%$ in this cohort was comparable to reported experiences during the pandemic [45-47]. Our study has limitations, including a low retained number of patients in the multivariable logistic regression in the provided model (409 out of $1468(27.82 \%)$ ). However, this low number depends on the clinically dependent variables utilized in the model that is limited to a small number of critically ill patients on ICU admission as the need for intubation and vasopressors.

\section{Conclusion}

Five clinical predictors of 28-day ICU outcome identified using DT algorithmic analysis of COVID-19 patients admitted to ICU. DT analysis may be used in ICU for early identification of critically ill COVID-19 patients who are at high risk of 28-day mortality. Further studies are required to validate these results.

\section{Declarations}

\section{Acknowledgment:}

The authors would like to thank the Saudi Critical Care Trials Group for their kind guidance, and the Saudi COVID working group for their marvelous help in data sharing and collection including, Mohamed Ali AlOdat, Huda A Mhawish, Bobby Rose Marasigan, Armalene Cabuyadao Bautista, Katrina Baguisa, Zahra Alfrdan, Bernadette Daher, Mohammed H. Lhmdi, Waleed Alatreeby, Mahdi Othman, Ashraf Shawat, Ahmed Y Zakarya, Mohamed Abdulzaher, Saleh Samir Saleh Ashgar, Jumana Khalid Nabeeh Alansari, Mohammed Amin, Mohammed Rio, Duaa Jihad Zain Basrawi, Hussam Mahmoud Omar Baeissa, Bashayer Mohammed Alsaadi, Ibrahim Mohamed Ibrahim Nasr, Mahdi Alajaj, Ozaz Hamza, Saud A Alghamdi, Hamza S Ghazal, Omar S Elrabi, Sumaih A Alomran, Saleh S Alomari, Taghred M Shwaish, Afaf S Alotaibi, Omar Alrabi, Amani Alharbi, Mohammed Alzanbaqi, Fahad Alsallum, Mohammed Bashanfar, Mohammed Jastaniah, Hamdan Alguydi, Ahmed Fakhri, Sheryl S. Lugue. Their willingness to give their time so generously has been very much appreciated.

Authors' contributions: Design conception: AE, HS, AR. Data collection: AE, HS, AO, AM, AG, AT, M.H.A, AS, AK, ZA, GM, WT, S.A.A, FF, AH, JT R.G.M and the Saudi COVID working group. Data validation and cleaning: $A E, H S$, and HM. Statistical analysis, model development, and Validation: HM. Acquisition, analysis, or interpretation of data: AE. AR and HM. Drafting and writing of the manuscript: AE \& AR. Editing and reviewing: YA, WH, MS, ZA, AO, and HM. Critical revision of the manuscript for important intellectual content, final review, and approval: all authors.

Funding: No funding

Competing interests: All authors disclosed no conflict of interest related to this work. 
Availability of data and material: Alyaa Elhazmi, Hend Sallam, and Hani Mufti has full access to all the data in the study and take responsibility for the integrity of the data, and the accuracy of the analysis and are willing to submit to an external review of the data upon request.

Ethics approval: Approval was obtained from the Central Institutional Review Board at the Saudi Ministry of Health approved this study [20-80E]. Individual ethical board approvals of the participating centers were obtained.

Consent to participate was waived as all data are unidentified.

Consent for publication all authors accept and confirm publication.

\section{References}

1. Zhu N, Zhang D, Wang W, et al. A Novel Coronavirus from Patients with Pneumonia in China, 2019. New England Journal of Medicine 2020;382(8):727-733. https://doi.org/10.1056/NEJMoa2001017

2. Cucinotta D, Vanelli M. WHO Declares COVID-19 a Pandemic. Acta Biomed 2020;91(1):157-160. (In eng). https://doi.org/10.23750/abm.v91i1.9397

3. Ruan Q, Yang K, Wang W, Jiang L, Song J. Clinical predictors of mortality due to COVID-19 based on an analysis of data of 150 patients from Wuhan, China. Intensive Care Med 2020;46(5):846-848. (In eng). https://doi.org/10.1007/s00134-020-05991-x

4. Ferrando C, Suarez-Sipmann F, Mellado-Artigas R, et al. Clinical features, ventilatory management, and outcome of ARDS caused by COVID-19 are similar to other causes of ARDS. Intensive care medicine 2020;46(12):2200-2211. https://doi.org/10.1007/s00134-020-06192-2

5. Rochwerg B, Einav S, Chaudhuri D, et al. The role for high flow nasal cannula as a respiratory support strategy in adults: a clinical practice guideline. Intensive care medicine 2020;46(12):2226-2237. https://doi.org/10.1007/s00134-020-06312-y

6. Helms J, Tacquard C, Severac F, et al. High risk of thrombosis in patients with severe SARS-CoV-2 infection: a multicenter prospective cohort study. Intensive care medicine 2020;46(6):1089-1098. https://doi.org/10.1007/s00134-020-06062-x

7. Joly BS, Siguret $V$, Veyradier A. Understanding pathophysiology of hemostasis disorders in critically ill patients with COVID-19. Intensive care medicine 2020;46(8):1603-1606. https://doi.org/10.1007/s00134-020-06088-1

8. Matthay MA, Arabi YM, Siegel ER, et al. Phenotypes and personalized medicine in the acute respiratory distress syndrome. Intensive care medicine 2020:1-17. https://doi.org/10.1007/s00134020-06296-9

9. Karthikeyan A, Garg A, Vinod P, Priyakumar UD. Machine learning based clinical decision support system for early COVID-19 mortality prediction. Frontiers in public health 2021;9. https://doi.org/10.1007/s00134-020-06296-9 
10. Read K, LaPolla FWZ. A new hat for librarians: providing REDCap support to establish the library as a central data hub. J Med Libr Assoc 2018;106(1):120-126. https://doi.org/10.5195/jmla.2018.327

11. Vincent JL, Moreno R, Takala J, et al. The SOFA (Sepsis-related Organ Failure Assessment) score to describe organ dysfunction/failure. On behalf of the Working Group on Sepsis-Related Problems of the European Society of Intensive Care Medicine. Intensive Care Med 1996;22(7):707-10. (In eng). https://doi.org/10.1007/bf01709751

12. Witten IH, F.E., Hall MA., Data mining: practical machine learning tools and techniques. San Francisco: Morgan Kaufmann Publishers; , 2011.

13. J. Ross, Q., C4.5: Programs for Machine Learning, . Morgan Kaufmann Publishers Inc., San Francisco, CA, USA, 1993.Organization. https://apps.who.int/iris/handle/10665/258695. License: CC BY-NC-SA 3.0 IGO.

14. Gu Q, Zhu L, Cai Z. Evaluation Measures of the Classification Performance of Imbalanced Data Sets. 2009:461. https://doi.org/10.1007/978-3-642-04962-0_53

15. Arabi YM, Azoulay E, Al-Dorzi HM, et al. How the COVID-19 pandemic will change the future of critical care. Intensive Care Med 2021;47(3):282-291. https://doi.org/10.1007/s00134-021-06352-y

16. de Lange DW, Soares M, Pilcher D. ICU beds: less is more? No. Intensive Care Medicine 2020;46(8):1597-1599. https://doi.org/10.1007/s00134-020-06089-0

17. Vincent J-L, Creteur J. Ethical aspects of the COVID-19 crisis: How to deal with an overwhelming shortage of acute beds. European Heart Journal Acute Cardiovascular Care 2020;9(3):248-252. https://doi.org/10.1177/2048872620922788

18. Li S, Lin Y, Zhu T, et al. Development and external evaluation of predictions models for mortality of COVID-19 patients using machine learning method. Neural Computing and Applications 2021:1-10. https://doi.org/10.1007/s00521-020-05592-1

19. Xu Q, Zhan X, Zhou Z, et al. Al-based analysis of CT images for rapid triage of COVID-19 patients. NPJ Digit Med 2021;4(1):75. (In eng). https://doi.org/10.1038/s41746-021-00446-z

20. Mahdjoub E, Mohammad W, Lefevre T, Debray MP, Khalil A. Admission chest CT score predicts 5-day outcome in patients with COVID-19. Intensive Care Med 2020;46(8):1648-1650. (In eng). https://doi.org/10.1007/s00134-020-06118-y

21. Giangiuliani G, Mancini A, Gui D. Validation of a severity of illness score (APACHE II) in a surgical intensive care unit. Intensive Care Med 1989;15(8):519-22. (In eng). https://doi.org/10.1007/bf00273563

22. Cho DY, Wang YC. Comparison of the APACHE III, APACHE II and Glasgow Coma Scale in acute head injury for prediction of mortality and functional outcome. Intensive Care Med 1997;23(1):77-84. (In eng). https://doi.org/10.1007/s001340050294

23. Capuzzo M, Valpondi V, Sgarbi A, et al. Validation of severity scoring systems SAPS II and APACHE II in a single-center population. Intensive Care Med 2000;26(12):1779-85.

https://doi.org/10.1007/s001340000715

Page 18/23 
24. Knight SR, Ho A, Pius R, et al. Risk stratification of patients admitted to hospital with covid-19 using the ISARIC WHO Clinical Characterisation Protocol: development and validation of the 4C Mortality Score. bmj 2020;370. https://doi.org/10.1136/bmj.m3339

25. Polderman KH, Jorna EM, Girbes AR. Inter-observer variability in APACHE II scoring: effect of strict guidelines and training. Intensive Care Medicine 2001;27(8):1365-1369. https://doi.org/10.1007/s001340101012

26. Shipe ME, Deppen SA, Farjah F, Grogan EL. Developing prediction models for clinical use using logistic regression: an overview. J Thorac Dis 2019;11(Suppl 4):S574-s584. (In eng). https://doi.org/10.21037/jtd.2019.01.25

27. Ahmad LG, Eshlaghy A, Poorebrahimi A, Ebrahimi M, Razavi A. Using three machine learning techniques for predicting breast cancer recurrence. J Health Med Inform 2013;4(124):3. https://doi.org/10.4172/2157-7420.1000124

28. Xiao Y, Wu J, Lin Z, Zhao X. A deep learning-based multi-model ensemble method for cancer prediction. Computer methods and programs in biomedicine 2018;153:1-9. https://doi.org/10.1016/j.cmpb.2017.09.005

29. Mihaylov I, Nisheva M, Vassilev D. Application of machine learning models for survival prognosis in breast cancer studies. Information 2019;10(3):93. https://doi.org/10.3390/info10030093

30. Maclin PS, Dempsey J, Brooks J, Rand J. Using neural networks to diagnose cancer. J Med Syst 1991;15(1):11-9. (In eng). https://doi.org/10.1007/bf00993877

31. Simes RJ. Treatment selection for cancer patients: application of statistical decision theory to the treatment of advanced ovarian cancer. Journal of chronic diseases 1985;38(2):171-186. https://doi.org/10.1016/0021-9681(85)90090-6

32. Bishop C. (2006) Pattern Recognition and Machine Learning (Information Science and Statistics) (Springer-Verlag New York, Inc., Secaucus, NJ, USA).

33. Shabas $L$. Training algorithms for the decision tree method of pattern recognition. Cybernetics 1976;12(3):409-416. https://doi.org/10.1007/BF01070022

34. Kuncheva LI. ( 2014 )Combining pattern classifiers: methods and algorithms: John Wiley \& Sons, https://doi.org/10.1002/9781118914564

35. Trevor H, Robert T, Jerome F. Hastie T, Friedman J, Tibshirani R. (2009) The elements of statistical learning. Vol. 2. Springer;

36. Han J, Pei J, Kamber M. (2011) Data mining: concepts and techniques: Elsevier.

37. Danwang C, Endomba FT, Nkeck JR, Wouna DLA, Robert A, Noubiap JJ. A meta-analysis of potential biomarkers associated with severity of coronavirus disease 2019 (COVID-19). Biomarker research 2020;8(1):1-13. https://doi.org/10.1186/s40364-020-00217-0

38. Williamson EJ, Walker AJ, Bhaskaran K, et al. Factors associated with COVID-19-related death using OpenSAFELY. Nature 2020;584(7821):430-436. https://doi.org/10.1038/s41586-020-2521-4 
39. Zanella A, Florio G, Antonelli M, et al. Time course of risk factors associated with mortality of 1260 critically ill patients with COVID-19 admitted to 24 Italian intensive care units. Intensive care medicine 2021:1-14.

40. Du P, Li D, Wang A, Shen S, Ma Z, Li X. A Systematic Review and Meta-Analysis of Risk Factors Associated with Severity and Death in COVID-19 Patients. Can J Infect Dis Med Microbiol 2021;2021:6660930. https://doi.org/10.1155/2021/6660930

41. Yang Q, Li J, Zhang Z, et al. Clinical characteristics and a decision tree model to predict death outcome in severe COVID-19 patients. BMC Infectious Diseases 2021;21(1):783. https://doi.org/10.1186/s12879-021-06478-w

42. Yan $\mathrm{L}$, Zhang $\mathrm{H}$, Goncalves J, et al. A machine learning-based model for survival prediction in patients with severe COVID-19 infection. medRxiv. March 2020;17. https://doi.org/10.1101/2020.02.27.20028027

43. Toraih EA, Elshazli RM, Hussein $\mathrm{MH}$, et al. Association of cardiac biomarkers and comorbidities with increased mortality, severity, and cardiac injury in COVID-19 patients: a meta-regression and decision tree analysis. Journal of medical virology 2020;92(11):2473-2488. https://doi.org/10.1002/jmv.26166

44. Armstrong RA, Kane AD, Kursumovic E, Oglesby FC, Cook TM. Mortality in patients admitted to intensive care with COVID-19: an updated systematic review and meta-analysis of observational studies. Anaesthesia 2021;76(4):537-548. https://doi.org/10.1111/anae.15425.

45. Almazeedi S, Al-Youha S, Jamal MH, et al. Characteristics, risk factors and outcomes among the first consecutive 1096 patients diagnosed with COVID-19 in Kuwait. EClinicalMedicine 2020;24:100448. (In eng). https://doi.org/10.1016/j.eclinm.2020.100448.

46. Lee JS, Godard A. Critical care for COVID-19 during a humanitarian crisis-lessons learnt from Yemen. Crit Care 2020;24(1):572. (In eng). https://doi.org/10.1186/s13054-020-03281-y.

47. Shahriarirad R, Khodamoradi Z, Erfani A, et al. Epidemiological and clinical features of 2019 novel coronavirus diseases (COVID-19) in the South of Iran. BMC Infect Dis 2020;20(1):427. (In eng). https://doi.org/10.1186/s12879-020-05128-x

\section{Figures}


KSA COVID-19 ICU Cummulative Incidance of Mortality

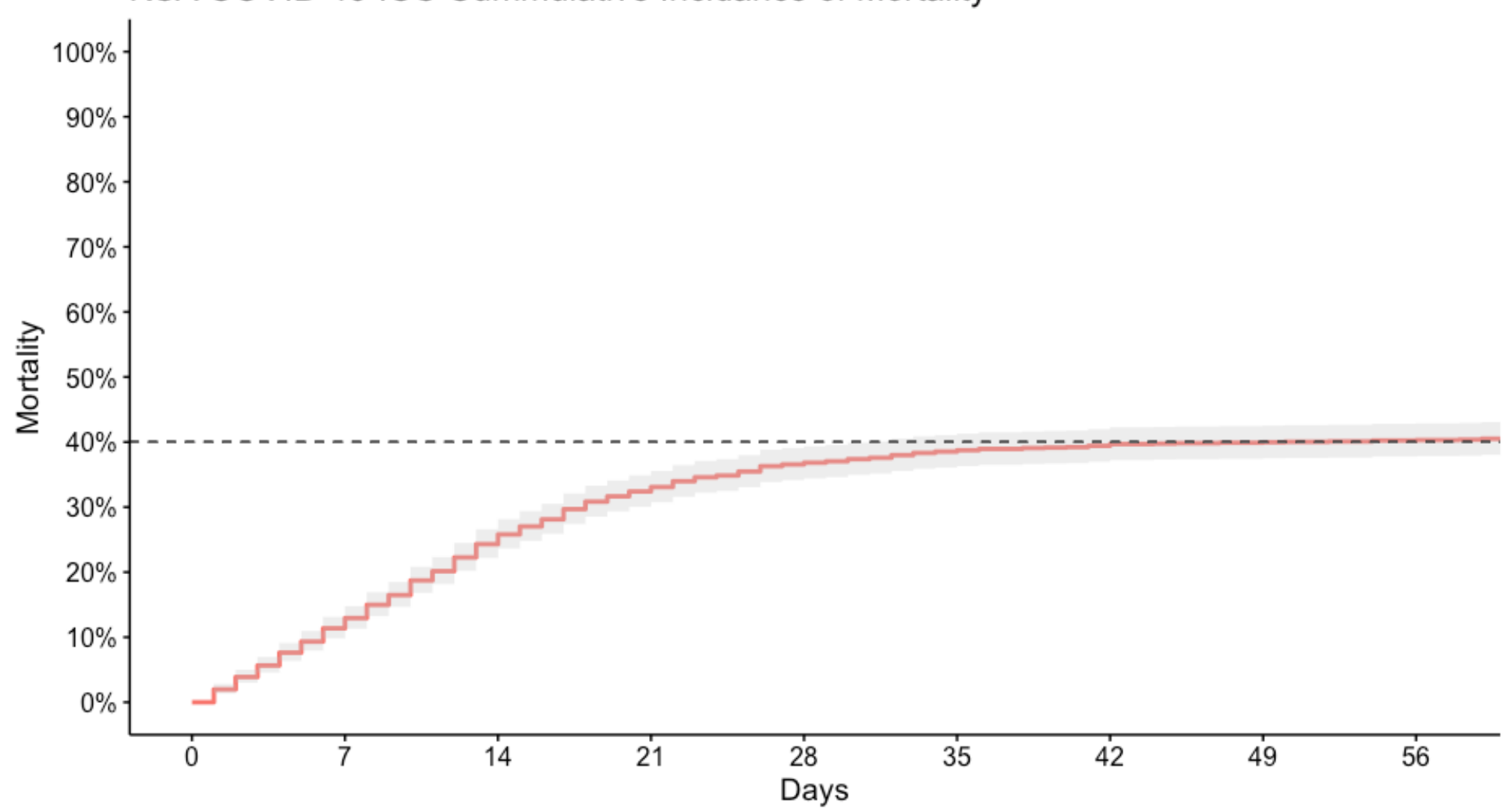

Number at risk

$\begin{array}{llllllllll}\text { All } & 1469 & 1302 & 1112 & 993 & 932 & 903 & 890 & 883 & 878\end{array}$

\section{Figure 1}

Kaplan Maier curve for COVID-19 cumulative incidence of mortality. 


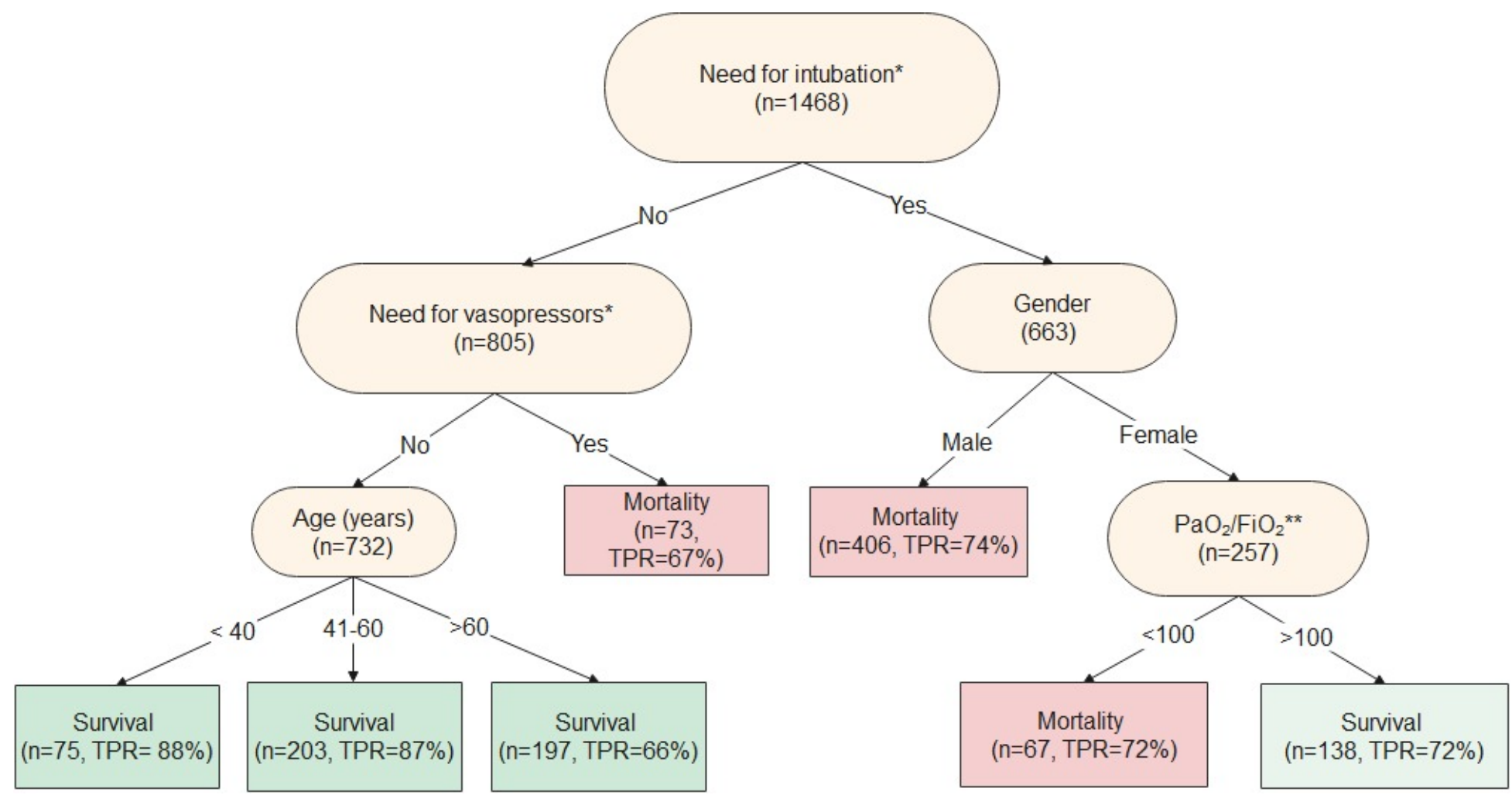

\section{Figure 2}

Decision Tree (DT) algorithm for predictors of mortality. - n: number of subjects in the leaf, TPR: true positive rate (sensitivity). - *During five days of ICU admission - ** In the first 24 hours of ICU admission. DT algorithm with ROC-AUC= $75.42 \%$ (95\% Cl= 74.84-78.95). - Number of patients retained in the model= 1043 out of 1468 (71.04\%). - The need for intubation during the first five days of ICU admission was the first splitting variable (Root node); in those who required intubation, gender was the next splitting step. If the patient was a Male, a high probability of mortality with $74 \%$ accuracy is anticipated. While if female gender, the $\mathrm{PaO} 2 / \mathrm{FiO} 2$ ratio $<100$ had a high probability of mortality with an accuracy of $72 \%$. On the other hand, for those who did not require intubation nor vasopressors during the first five days, age affected the outcome widely (e.g., high probability of survival with an $88 \%$ accuracy in age group less than 40). 


\begin{tabular}{|c|c|c|c|c|}
\hline Variable & & Odds ratio & & $\mathbf{p}$ \\
\hline \multirow[t]{3}{*}{ Age [Years] } & $<41$ & $\mathbf{v}$ & Reference & \\
\hline & $41-60$ & $\mapsto$ & $1.26(0.76,2.09)$ & 0.376 \\
\hline & $>60$ & $\mapsto$ & $2.52(1.46,4.41)$ & 0.001 \\
\hline \multirow[t]{2}{*}{ Gender } & Female & & Reference & \\
\hline & Male & - & $1.96(1.34,2.89)$ & $<0.001$ \\
\hline \multirow[t]{2}{*}{ DM } & No & & Reference & \\
\hline & Yes & † & $0.83(0.57,1.19)$ & 0.307 \\
\hline \multirow[t]{2}{*}{ IHD } & No & & Reference & \\
\hline & Yes & $\mapsto$ & $1.31(0.79,2.15)$ & 0.291 \\
\hline \multirow[t]{2}{*}{ Respiratory component of SOFA score (PaO2/FiO2) } & Category 0 - 3 & & Reference & \\
\hline & Category 4 & & $1.44(1.04,2.01)$ & 0.029 \\
\hline \multirow[t]{2}{*}{ Need for vasopressors/inotropes } & No & & Reference & \\
\hline & Yes & 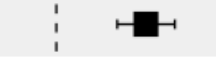 & $3.15(2.18,4.56)$ & $<0.001$ \\
\hline \multirow[t]{2}{*}{ Need for Intubation in the ICU } & No & & Reference & \\
\hline & Yes & 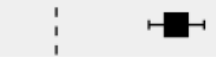 & $4.64(3.27,6.63)$ & $<0.001$ \\
\hline \multirow[t]{2}{*}{ NL Ratio } & $\leq 8.5$ & $\varphi$ & Reference & \\
\hline & $>8.5$ & H & $1.65(1.19,2.30)$ & 0.003 \\
\hline
\end{tabular}

\section{Figure 3}

Stepwise logistic regression of 28-day mortality $\mathrm{PaO} 2 / \mathrm{FiO2}$, Partial pressure of oxygen to fraction of inspired oxygen ratio. DM: Diabetes mellitus. IHD: Ischemic heart disease, LDH: lactate dehydrogenase. Stepwise Logistic Regression: ROC-AUC= 79.96\% (95\% Cl= 76.91-83.02). Number of patients retained in the model $=843$ Out of $1468(57.35 \%)$ 